\title{
VAC_03 - Streptococcus agalactiae prevalent capsular types and impact of capsular-based vaccines in pregnant women population in Rio de Janeiro
}

\author{
Rosana Rocha Barros ${ }^{1 *}$; Karen Baeta Alves ${ }^{1}$; Fernanda Baptista O Luiz ${ }^{1}$; Douglas Guedes Ferreira ${ }^{1}$. \\ ${ }^{1}$ UFF - Universidade Federal Fluminense.
}

Introduction: Streptococcus agalactiae (Group B Streptococcus - GBS) is a major cause of severe neonatal infections, such as septicemia and meningitis. The maternal vaginal colonization represents the most important risk factor for infection in the newborn, because microorganism can be vertically transmitted to the child during gestation or birth. Approximately $50 \%$ of neonates born from women colonized by GBS will also be colonized, with $1-2 \%$ developing early neonatal infection. Maternal immunization against GBS is a promising alternative to prevent neonatal infections. GBS capsule, a polysaccharide structure, with 10 described types (Ia, Ib, II-IX), is an important virulence factor, epidemiological marker and also represents the vaccine target at a more advanced stage of development. To date, a hexavalent vaccine, Ia, Ib, II, III, IV and V, conjugated to CRM197 carrier is under clinical trial. These capsular types correspond to the great majority of colonization and infection around the world, however, the prevalence of each type varies among isolates recovered in different regions. Therefore, it is fundamental to determine the predominant capsular types in each geographical area, generating local epidemiological data and evaluating the vaccine impact.

Objective: The objective of this study was to determine the distribution of capsular types of GBS recovered from pregnant women resident in the metropolitan area of Rio de Janeiro over a period of 16 years (March 2002-March 2018).

Methodology: The study included 124 GBS isolates (one isolate of each subject) recovered from vaginal secretion and urine specimens. Multiplex-PCR with specific primers to each type was performed to determine the capsular types.

Results: The most frequent types were Ia (33.1\%), II (25.8\%) and V (21.8\%). Other types found were Ib $(8.9 \%)$, III (8.9\%) and IV (1.6\%). While type Ia was prevalent during the whole period, fluctuations in distribution of other types, specially $\mathrm{V}$, were observed over time.

Conclusion: Distribution of maternal GBS capsular types in the region, with prevalence of Ia and II, is quite different from other parts of the world, where type III predominates in both infection and colonization isolates, being the most neonatal severe infections associated type. The knowledge about GBS capsular type distribution is essential to predict the impact of capsule-based vaccines in the local population. The hexavalent vaccine would have a $100 \%$ of theoretic impact in the population of pregnant women resident in the metropolitan area of Rio de Janeiro, however, a previous approach, a trivalent vaccine (Ia, Ib and III) that had also undergone to clinical trials, would have a low impact in such population.

Keywords: Streptococcus agalactiae; vaccine; capsular typing 